\title{
A Survey on Fuzzy Differences
}

\section{Francielle Santo Pedro ${ }^{1}$ Laécio Carvalho de Barros ${ }^{2}$ Luciana Takata Gomes $^{3}$}

\author{
${ }^{1}$ Department of Applied Mathematics, IMECC, State University of Campinas, Campinas, Brazil \\ ${ }^{2}$ Department of Applied Mathematics, IMECC, State University of Campinas, Campinas, Brazil \\ ${ }^{3}$ Dept. Physics, Chemistry and Mathematics, UFSCar, Federal University of São Carlos, Sorocaba, Brazil
}

\begin{abstract}
Several definitions of difference between fuzzy numbers are well established in literature: standard, Hukuhara, generalized Hukuhara, generalized, CIA and other differences based on joint possibility distributions. We present and compare them. An example of epidemiological model of a disease with direct transmission illustrates the different approaches. Finally, we briefly state some derivatives defined by using the cited differences.
\end{abstract}

Keywords: Fuzzy numbers, Differences, Derivatives

\section{Introduction}

The standard difference between two fuzzy numbers $A$ and $B$ is based on the difference between intervals [1]. For each $\alpha$ in the unit interval, it takes into account all the possible combinations between two elements, one from the $\alpha$-level of $A$ and the other from the $\alpha$-level of $B$. Consequently, the result is always greater (in diameter) than any of the sets involved in the operation. Thus, the difference between two non-crisp numbers is always a non-crisp number and subtracting a non-crisp number from itself is never the crisp number zero.

Using the Hukuhara difference [8], the result of subtracting a non-crisp number $A$ from itself $\left(A \ominus_{H} A\right)$ is, in fact, zero. However, for this case, a necessary condition for the subtraction between two different fuzzy numbers $A$ and $B$ to exist is the first term to have bigger diameter than the second one. Generalizing the idea of this difference, $[9,10]$ proposed the generalized Hukuhara difference, which also satisfies $A-{ }_{g H} A=0$ and is defined for a bigger class of fuzzy numbers than the Hukuhara difference. An extension of generalized Hukuhara difference is the generalized difference $[2,10]$, which has the same results of the generalized Hukuhara operator (when it exists), but is defined for more pairs of fuzzy numbers.

Another possibility is CIA (Constraint Interval Arithmetic) [7]. In this case, the diameter of the difference between two fuzzy numbers using CIA is smaller than using standard difference.

All differences mentioned above makes use of the interval arithmetic on $\alpha$-levels. Extensions to fuzzy numbers are drawn up via Stacking Theorem [3] over the resulting $\alpha$-levels.
Another way is similar to arithmetic for random variables, that is, the subtractions between fuzzy numbers are obtained using the joint possibility (or membership) distribution between the involved fuzzy numbers [5]. The comparison between the results obtained from two approaches is made via a kind of Nguyen's theorem extension [5].

In this study we present the definitions of all cited differences as well as its derivatives and we analyze its relations and exhibit an illustrative example.

\section{Preliminary}

In this section we present some basic concepts and notation used in this study.

Definition 2.1 A fuzzy subset $A$ of $U$ is characterized by a membership function $\mu_{A}: U \longrightarrow[0,1]$.

Since a classical set is defined by its characteristic function of counter-domain $\{0,1\}$, we can say that a classical set is a particular case of a fuzzy set. We denote by $\mathcal{F}(U)$ the family of all fuzzy subsets of $U$.

In what follows $U$ is as a topological space.

Definition 2.2 Let $A$ be a fuzzy subset of $U$ and $\alpha \in[0,1]$. The $\alpha$-level of $A$ is the classical subset of $U$ defined by

$$
[A]_{\alpha}=\left\{x \in U: \mu_{A}(x) \geq \alpha\right\} \text { for } 0<\alpha \leq 1 .
$$

The zero level of a fuzzy set $A$ of $U$ is defined as the smallest closed set that contain the support of the fuzzy set $A$, that is, $[A]_{0}=\operatorname{cl}\left\{x \in U: \mu_{A}(x)>0\right\}$.

The family of all fuzzy subsets of $U$ with nonempty, compact and convex $\alpha$-levels will be denoted by $\mathcal{F}_{\mathcal{C}}(U)$.

Definition 2.3 A fuzzy subset $A$ is said to be a fuzzy number when its universe is the set of real numbers $\mathbb{R}$ and satisfy:

i. All $\alpha$-levels of $A$ are nonempty closed intervals of $\mathbb{R}$;

ii. $\left\{x \in U: \mu_{A}(x)>0\right\}$ is a limited set.

The family of fuzzy numbers coincides with $\mathcal{F}_{\mathcal{C}}(\mathbb{R})$ and will be denoted by $\mathbb{R}_{\mathcal{F}}$.

Theorem 2.4 (Stacking Theorem, [3, 11]) If $A$ is a fuzzy number and $[A]_{\alpha}$ are its level-sets then: 
i. $[A]_{\alpha}$ is a closed interval $[A]_{\alpha}=\left[a_{\alpha}^{-}, a_{\alpha}^{+}\right]$, for any $\alpha \in[0,1]$;

ii. If $0 \leq \alpha_{1} \leq \alpha_{2} \leq 1$, then $[A]_{\alpha_{2}} \subseteq[A]_{\alpha_{1}}$.

iii. For any sequence $\alpha_{n}$ which converges from below to $\alpha \in(0,1]$ we have

$$
\bigcap_{n=1}^{\infty}[A]_{\alpha_{n}}=[A]_{\alpha} .
$$

iv For any sequence $\alpha_{n}$ which converges from above to 0 we have

$$
c l\left(\bigcup_{n=1}^{\infty}[A]_{\alpha_{n}}\right)=[A]_{0} .
$$

Definition 2.5 $A$ t-norm $T$ is any binary operation $T:[0,1] \times[0,1] \rightarrow[0,1]$ that has the following properties:

1. neutral element: $T(1, x)=x$;

2. commutativity: $T(x, y)=T(y, x)$;

3. associativity: $T(x, T(y, z))=T(T(x, y), z)$;

4. monotonicity: if $x \leq u$ and $y \leq v$, then $T(x, y) \leq T(u, v)$.

The following concepts are from the possibility theory and will be used to define the interactive difference between fuzzy numbers [5].

Definition 2.6 Let $A$ and $B$ be fuzzy numbers and $J \in \mathcal{F}_{\mathcal{C}}\left(\mathbb{R}^{2}\right)$. Then $J$ is a joint possibility distribution of $A$ and $B$ if

$\max _{y \in \mathbb{R}} \mu_{J}(x, y)=\mu_{A}(x) \quad$ and $\quad \max _{x \in \mathbb{R}} \mu_{J}(x, y)=\mu_{B}(y)$.

Moreover, $\mu_{A}$ and $\mu_{B}$ are called marginal distributions of $J$.

If the joint possibility distribution is given by a $t$ norm $T$, we have

$$
\mu_{J}(x, y)=T\left(\mu_{A}(x), \mu_{B}(y)\right) .
$$

When $T=\min , A$ and $B$ are called non-interactive fuzzy numbers. The following statement is a more general definition that has the Zadeh's extension as a particular case when the $t$-norm is the minimum norm.

Definition 2.7 Let $J$ be a joint possibility distribution with marginal possibility distributions $\mu_{A}$ and $\mu_{B}$, and let $f: \mathbb{R}^{2} \longrightarrow \mathbb{R}$ be a function. Then the extension of $f$ by $J$ is the function $f_{J}$ whose membership function is given by

$$
\mu_{f_{J}(A, B)}(z)=\left\{\begin{array}{ccc}
\sup _{y=f(x, y)} \mu_{J}(x, y) & \text { if } & f^{-1}(z) \neq \emptyset \\
0 & \text { if } & f^{-1}(z)=\emptyset
\end{array}\right.
$$

where $f^{-1}(z)=\{(x, y): f(x, y)=z\}$.

The Zadeh's extension principle is an important tool widely used to extend point-valued functions to fuzzy-set-valued functions.
Theorem 2.8 [5] Let $A, B \in \mathcal{F}(\mathbb{R})$ be completely correlated fuzzy numbers, $J$ its joint possibility distribution and $f: \mathbb{R}^{2} \longrightarrow \mathbb{R}$ a continuous function. Then,

$$
\left[f_{J}(A, B)\right]_{\alpha}=f\left([J]_{\alpha}\right) .
$$

Definition 2.9 Two fuzzy numbers $A$ and $B$ are said to be completely correlated if there are $q, r \in \mathbb{R}$, with $q \neq 0$, such that their joint possibility distribution $C$ is defined by

$$
\begin{aligned}
\mu_{C}(x, y) & =\mu_{A}(x) \mathcal{X}_{\{q x+r=y\}}(x, y) \\
& =\mu_{B}(y) \mathcal{X}_{\{q x+r=y\}}(x, y)
\end{aligned}
$$

where

$$
\mathcal{X}_{\{q x+r=y\}}(x, y)=\left\{\begin{array}{lll}
1 & \text { if } & q x+r=y \\
0 & \text { if } & q x+r \neq y
\end{array}\right.
$$

is the membership function of the real line $\{(x, y) \in$ $\left.\mathbb{R}^{2}: q x+r=y\right\}$.

\section{Difference between fuzzy numbers}

Next we present different manners (in literature) to realize the difference between fuzzy numbers.

\subsection{Difference via interval theory}

Initially we present the fuzzy differences arising from the interval theory.

Definition 3.1 (Standard difference) Let $A, B$ be fuzzy numbers with $\alpha$-levels given, respectively, by $\left[a_{\alpha}^{-}, a_{\alpha}^{+}\right]$and $\left[b_{\alpha}^{-}, b_{\alpha}^{+}\right]$. The $\alpha$-levels of standard difference, $A-B$, are defined by

$$
[A-B]_{\alpha}=\left[a_{\alpha}^{-}-b_{\alpha}^{+}, a_{\alpha}^{+}-b_{\alpha}^{-}\right] .
$$

Standard difference can also be called Minkowski difference.

In [6], the author proposed an arithmetic called CIA. For that goal, he redefined intervals as functions with real values, that is, an interval $\left[a^{-}, a^{+}\right]$ is given by the function $A^{I}\left(a^{-}, a^{+}, \lambda_{A}\right)=\{a: a=$ $\left.\left(1-\lambda_{A}\right) a^{-}+\lambda_{A} a^{+}, 0 \leq \lambda_{A} \leq 1\right\}$.

Definition 3.2 (CIA) The subtraction between two intervals $A=\left[a^{-}, a^{+}\right]$and $B=\left[b^{-}, b^{+}\right]$is given by

$$
\begin{aligned}
A-{ }_{C I A} B= & \left\{\left[\left(1-\lambda_{A}\right) a^{-}+\lambda_{A} a^{+}\right]-\left[\left(1-\lambda_{B}\right) b^{-}+\lambda_{B} b^{+}\right],\right. \\
& \left.\leq \lambda_{A} \leq 1,0 \leq \lambda_{B} \leq 1\right\} .
\end{aligned}
$$

Lodwick et. al [7] extended this idea for the fuzzy case from Theorem 2.4. Also, from this theorem it is possible to define Hukuhara difference between fuzzy numbers.

Definition 3.3 (CIA) The subtraction between two fuzzy numbers $A$ and $B$ is defined level-wise by

$$
\begin{gathered}
{\left[A-C_{C I A} B\right]_{\alpha}=\left\{\left[\left(1-\lambda_{A}\right) a_{\alpha}^{-}+\lambda_{A} a_{\alpha}^{+}\right]-\left[\left(1-\lambda_{B}\right) b_{\alpha}^{-}+\lambda_{B} b_{\alpha}^{+}\right],\right.} \\
\left.0 \leq \lambda_{A} \leq 1,0 \leq \lambda_{B} \leq 1\right\} .
\end{gathered}
$$


Remark 3.4 In the case where the two fuzzy numbers are the same, we have

$$
\begin{gathered}
{\left[A-{ }_{C I A} A\right]_{\alpha}=} \\
\left\{\left[\left(1-\lambda_{A}\right) a_{\alpha}^{-}+\lambda_{A} a_{\alpha}^{+}\right]-\left[\left(1-\lambda_{A}\right) a_{\alpha}^{-}+\lambda_{A} a_{\alpha}^{+}\right]\right\}=\{0\}
\end{gathered}
$$$$
\text { where } 0 \leq \lambda_{A} \leq 1 \text {. Therefore, } A-_{\text {CIA }} A=\{0\} \text {. }
$$

Definition 3.5 Given two fuzzy numbers $A, B$ the Hukuhara difference (H-difference) $A \ominus_{H} B=C$ is the fuzzy number $C$ such that $A=B+C$, if it exists.

Definition 3.6 [9, 10] Given two fuzzy numbers $A, B$ the generalized Hukuhara difference ( $g H$ difference) $A \ominus_{g H} B=C$ is the fuzzy number $C$ (if it exists) such that

$$
\begin{cases}\text { (i) } & A=B+C \quad \text { or } \\ \text { (ii) } \quad B=A-C .\end{cases}
$$

Definition 3.7 [2, 10] Given two fuzzy numbers $A, B$ the generalized difference ( $g$-difference) $A \ominus_{g}$ $B=C$ is the fuzzy number $C$ with $\alpha$-levels

$$
\left[A \ominus_{g} B\right]_{\alpha}=c l \bigcup_{\beta \geq \alpha}\left([A]_{\beta} \ominus_{g H}[B]_{\beta}\right), \forall \alpha \in[0,1]
$$

where the $g H$-difference $\left(\ominus_{g H}\right)$ is related to the intervals $[A]_{\beta}$ and $[B]_{\beta}$.

In $[2,10]$ the generalized difference between fuzzy numbers was proposed as a difference that always exists and results in a fuzzy number. But for this, as observed in [12], a convexification is required for the difference to be always a fuzzy number.

Each of the differences presented in this subsection, $A-B$ is a fuzzy numbers according to Theorem 2.4 .

\subsection{Differences via joint possibility distribution}

Differences via joint possibility distribution are obtained with the help of Definition 2.7. Note that this form of dealing with fuzzy numbers is totally inspired by the arithmetic for random variables, which considers the joint probability distribution.

Definition 3.8 Suppose $A$ and $B$ are two fuzzy numbers. Let $f: \mathbb{R}^{2} \rightarrow \mathbb{R}$ be defined by $f(x, y)=$ $x-y$, that is, the subtraction operator for real numbers. The difference by joint distribution $J$ is the fuzzy number $A-{ }_{J} B$, whose membership function is defined by

$\mu_{\left(A-{ }_{J} B\right)}(z)=\left\{\begin{array}{l}\sup _{f^{-1}(z)} \mu_{J}(x, y) \quad \text { if } \quad f^{-1}(z) \neq \emptyset\end{array}\right.$

where $f^{-1}(z)=\{(x, y): f(x, y)=x-y=z\}$.

Next the difference with joint possibility distribution is given by t-norms.
Definition 3.9 (Difference by t-norm) Let $A, B$ be fuzzy numbers and $f(x, y)=x-y$ the subtraction operator, then the extension sup $-T$ of the fuzzy number $A-_{T} B$ is obtained by the following membership function

$$
\mu_{A-B}(z)=\sup _{x-y} T\left(\mu_{A}(x), \mu_{B}(y)\right), z \in \mathbb{R} .
$$

Definition 3.10 The Zadeh difference between two fuzzy numbers is the fuzzy number $A-B$, whose membership function is given by

$$
\begin{gathered}
\mu_{(A-B)}(z)=\left\{\sup _{f^{-1}(z)}\left\{\min \left[\mu_{A}(x), \mu_{B}(y)\right]\right\},\right. \\
\text { where } f^{-1}(z)=\{(x, y): f(x, y)=x-y=z\} .
\end{gathered}
$$

That is, the Zadeh difference consider $A$ and $B$ noninteractive, since the join possibility distribution is given by minimum $t$-norm.

Proposition 3.11 Let $A, B$ be fuzzy numbers with $\alpha$-levels given, respectively, by $\left[a_{\alpha}^{-}, a_{\alpha}^{+}\right]$and $\left[b_{\alpha}^{-}, b_{\alpha}^{+}\right]$. Then the $\alpha$-levels of Zadeh difference are given by

$$
[A-B]_{\alpha}=\left[a_{\alpha}^{-}-b_{\alpha}^{+}, a_{\alpha}^{+}-b_{\alpha}^{-}\right]
$$

that is, the Zadeh difference coincides with the standard difference.

The proof is found in [16].

The difference using joint possibility distributions may not necessarily be given by t-norms.

Definition 3.12 [5] The subtraction of two completely correlated fuzzy numbers $A$ and $B$ is defined by

$$
\mu_{A-{ }_{C} B}(z)=\sup _{z=x-y} \mu_{C}(x, y)
$$

That is, $\mu_{A-{ }_{C} B}(z)=\sup _{z=x-y} \mu_{B}(y) \mathcal{X}_{\{q x+r=y\}}(x, y)$.

From Theorem 2.8, [5] proof that, for all $\alpha \in[0,1]$, we have

$$
\left[A-{ }_{C} B\right]_{\alpha}=(q-1)[B]_{\alpha}+r .
$$

Remark 3.13 The sum of two completely correlated fuzzy numbers $A$ and $B$ is given by

$$
\left[A+_{C} B\right]_{\alpha}=(q+1)[B]_{\alpha}+r .
$$

Proposition 3.14 Given two completely correlated fuzzy numbers $A$ and $B$ such that $[A]_{\alpha}=q[B]_{\alpha}+r$, the difference $A-{ }_{C} B$ has the following expression $[A-C B]_{\alpha}=$

$$
\left\{\begin{array}{ccc}
\text { i. } & {\left[(q-1) b_{\alpha}^{-},(q-1) b_{\alpha}^{+}\right]+r} & \text { if } q>1 \\
\text { ii. } & {\left[(q-1) b_{\alpha}^{+},(q-1) b_{\alpha}^{-}\right]+r} & \text { if } 0 \leq q \leq 1 \\
\text { iii. } & {\left[(q-1) b_{\alpha}^{+}+r,(q-1) b_{\alpha}^{-}+r\right]} & \text { if } q<0 .
\end{array}\right.
$$




\subsection{Comparing the differences}

Before we compare the different fuzzy differences we define the following notation: $X_{\text {difference }}$ is the set of the pairs of fuzzy numbers such that the difference between them exists.

For the difference diameters (diam) it is possible to show, by definition, that:

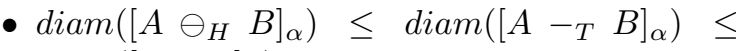
$\operatorname{diam}\left([A-B]_{\alpha}\right)$

- $\operatorname{diam}\left(\left[A \ominus_{g H} B\right]_{\alpha}\right) \leq \operatorname{diam}\left(\left[A-_{T} B\right]_{\alpha}\right) \leq$ $\operatorname{diam}\left([A-B]_{\alpha}\right)$

- $\operatorname{diam}\left(\left[\begin{array}{lll}A \ominus_{g} B & B\end{array}\right]_{\alpha} \leq \operatorname{diam}\left(\left[\begin{array}{lll}A & -T & B\end{array}\right]_{\alpha}\right) \leq\right.$ $\operatorname{diam}\left([A-B]_{\alpha}\right)$

- $\operatorname{diam}\left(\left[A-{ }_{J} B\right]_{\alpha}\right) \leq \operatorname{diam}\left([A-B]_{\alpha}\right)$;

- $\operatorname{diam}\left(\left[A-{ }_{C} B\right]_{\alpha}\right) \leq \operatorname{diam}\left([A-B]_{\alpha}\right)$;

- $\operatorname{diam}\left(\left[A-_{\mathrm{CIA}} B\right]_{\alpha}\right) \leq \operatorname{diam}\left([A-B]_{\alpha}\right)$.

Regarding the existence of each difference (which means that the result is a fuzzy number), we have:

- $X_{\text {standard }}, X_{\text {CIA }}, X_{T}, X_{J}$ and $X_{C}$ is equivalent to $\mathbb{R}_{\mathcal{F}} \times \mathbb{R}_{\mathcal{F}}$, that is, in these cases the difference between two fuzzy numbers always exists;

- $X_{T} \subseteq X_{\text {standard }}$;

- $X_{\mathrm{CIA}} \subset X_{\text {standard }}$;

- $X_{C} \subseteq X_{J} \subset X_{T}$;

- $X_{H} \subset X_{g H} \subset X_{g} \subset X_{T}$ and the differences $\mathrm{H}$ and $\mathrm{gH}$ do not always exist;

Remark 3.15 Note that the difference by joint possibility distribution always exists. The issue is to obtain this distribution.

As in the completely correlated difference, we have

$$
\left[A-{ }_{C} B\right]_{\alpha}=\left\{\begin{array}{ccc}
i . & {\left[a_{\alpha}^{-}-b_{\alpha}^{-}, a_{\alpha}^{+}-b_{\alpha}^{+}\right]} & \text {if } q \geq 1 \\
\text { ii. } & {\left[a_{\alpha}^{+}-b_{\alpha}^{+}, a_{\alpha}^{-}-b_{\alpha}^{-}\right]} & \text {if } 0 \leq q \leq 1 \\
\text { iii. } & {\left[a_{\alpha}^{-}-b_{\alpha}^{+}, a_{\alpha}^{+}-b_{\alpha}^{-}\right]} & \text {if } q<0 .
\end{array}\right.
$$

If $q \geq 1$ the difference $C$ is the Hukuhara difference. If $q \geq 0$ the difference $C$ is the generalized Hukuhara difference and for $q<0$ the difference $C$ is the standard difference. Comparing the completely correlated difference with $C I A$, we notice that they are equivalent if and only if $q=1$ when we compute $A-{ }_{C} A$ and $q<0$ if we compute $A-{ }_{C} B$ (see Figure 1).

The upside of using the joint possibility distributions is that it provides the membership function of the difference. On the other hand, differences via interval arithmetic present a practical method to calculate the difference. Also, they do not require knowledge of the joint possibility distribution.

\section{Application: Epidemiological Model}

The simplest classical model that describes the dynamics of transmitted diseases by direct contact without vital dynamics (i.e., without death/birth) is presented by the following equations [13]:

$$
\left\{\begin{array}{lll}
\frac{d S}{d t}=-\lambda S I ; & S(0)=S_{0}>0 \\
\frac{d I}{d t}=\lambda S I ; & I(0)=I_{0}>0,
\end{array}\right.
$$

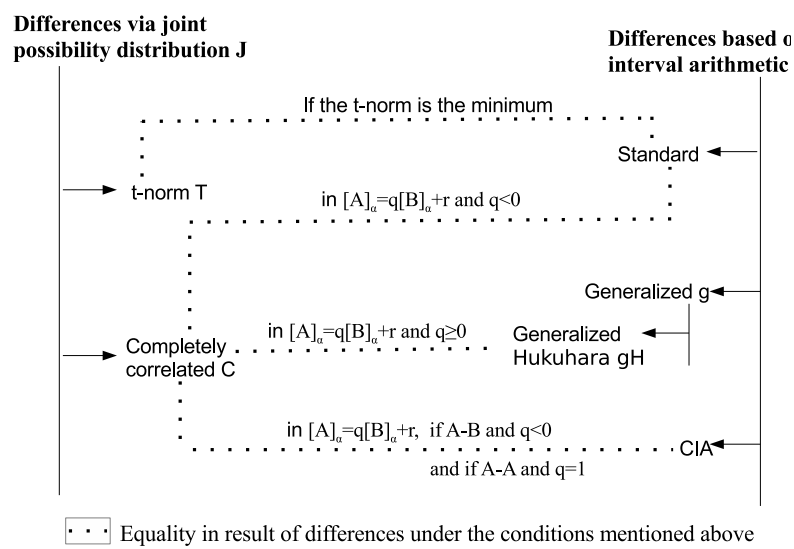

Figure 1: Relations between the differences defined by joint distribution and differences based on interval arithmetic. The dotted lines mean equality in the results of the subtractions between two fuzzy numbers (considering the mentioned hypothesis) .

where $S(t)$ and $I(t)$ are respectively the proportions of susceptible and infected individuals at instant $t$ and the parameter $\lambda>0$ is the disease transmission rate.

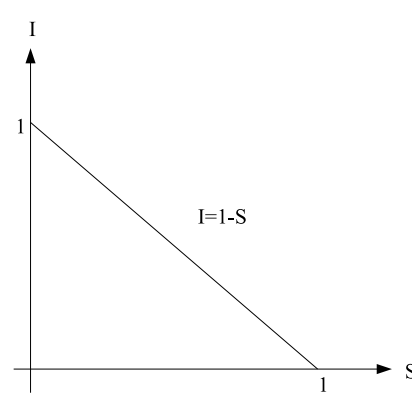

Figure 2: Proportion of susceptible individuals $(S)$ and infected ones $(I)$.

From (3), the sum of proportion of susceptible individuals $(S)$ and the proportion of infected ones $(I)$ is equal to 1 , that is,

$$
S(t)+I(t)=1, \quad \forall t \geq 0 .
$$

The solution of (3) is given by

$$
I(t)=\frac{I_{0} e^{\lambda t}}{S_{0}+I_{0} e^{\lambda t}} .
$$

Thus, from (4) it follows that

$$
S(t)=1-I(t)=\frac{S_{0}}{S_{0}+I_{0} e^{\lambda t}} .
$$

In the epidemiological point of view ( $S I$ model), once an individual is infected he/she never gets recovered. 
Now, if in (3) we consider that initial condition is uncertain, for each $t$, the solutions (5) and (6) are uncertain too. If we suppose that $S_{0}$ (and $I_{0}$ ) is a fuzzy triangular number, from (4) we have

$$
\left[S_{0}\right]_{\alpha}=\left[s_{\alpha}^{-}, s_{\alpha}^{+}\right]=[(b-a) \alpha+a,(b-c) \alpha+c],
$$

$0<a<b<c<1$ and $\left[I_{0}\right]_{\alpha}=1-\left[S_{0}\right]_{\alpha}$ will depend on which difference we use.

We compute $I_{0}$, using each one of the differences between 1 and $S_{0}$ given in the last section.

- If $S_{0}$ and $I_{0}$ are non-interactive, the difference between them is given by the standard form: $\left[I_{0}\right]_{\alpha}=\left[1-s_{\alpha}^{+}, 1-s_{\alpha}^{-}\right]$.

- If the difference is given by $T$-norms (different from the minimum $t$-norm), that is, $S_{0}$ and $I_{0}$ are interactive, we have $\left[I_{0}\right]_{\alpha}=\bigcup_{T(\xi, \eta) \geq \alpha}[1]_{\xi}-$ $\left[S_{0}\right]_{\eta}, \alpha \in(0,1]$.

- If $S_{0}$ and $I_{0}$ are completely correlated, we have $\left[I_{0}\right]_{\alpha}=1-\left[S_{0}\right]_{\alpha}$, with $q=-1$ and $r=1$, thus, $\left[I_{0}\right]_{\alpha}=\left[1-s_{\alpha}^{+}, 1-s_{\alpha}^{-}\right]$. In that case, from Remark 3.13, $\left[I_{0}\right]_{\alpha}+_{C}\left[S_{0}\right]_{\alpha}=1$.

- The Hukuhara difference does not exist, because we do not have an increasing diameter. On the other hand, the differences $\ominus_{g}$ and $\ominus_{g H}$, imply $\left[I_{0}\right]_{\alpha}=\left[1-s_{\alpha}^{+}, 1-s_{\alpha}^{-}\right]_{\alpha}$.

- Now, if we use $\left(-_{\text {CIA }}\right)$, we have $\left[I_{0}\right]_{\alpha}=\{1-$ $\left.\left[\left(1-\lambda_{S}\right) s_{\alpha}^{-}+\lambda s_{\alpha}^{+}\right], 0 \leq \lambda_{S} \leq 1\right\}=\{1-[(1-$ $\left.\left.\left.\lambda_{S}\right)((b-a) \alpha+a)+\lambda((b-c) \alpha+c)\right], 0 \leq \lambda_{S} \leq 1\right\}$ which is equivalent to the triangular number $(1-c ; 1-b ; 1-a)$.

Note that, $S_{\bar{t}}$ and $I_{\bar{t}}$ can not be triangular fuzzy numbers.

In summay, from the coherence point of view, the difference that better represents the $S I$ model is the completely correlated difference, because it is the one that best fits $S+I=1$, which faces the hypothesis of non existence of vital dynamic. Moreover, intuitively, the hypotheses $S+I=1$ suggests that there is interaction between $S$ and $I$. A study for this case we made in $[4,14]$.

The main reason for studying the difference between fuzzy numbers is due to connection with the derivatives.

\section{Fuzzy Derivative}

Different fuzzy differences produce different fuzzy derivatives for fuzzy functions. It is natural to expect that this derivative represents the various meanings of derivative. For example, the variation rate, since we expect that stationary systems (that is, constant function) must have null derivative. As we have seen, not always the difference between two identical fuzzy numbers is the crisp number zero (for example standard difference). For this reason, some difference are not indicate for to define derivative, since the division in the limit

$$
\lim _{h \rightarrow 0^{+}} \frac{F(x+h)-F(x)}{h}
$$

must be null, at least for the case where $F$ is constant fuzzy function. Wherein $F$ is a fuzzynumber-valued function, that is, fuzzy function of type $F:[a ; b] \rightarrow \mathbb{R}_{\mathcal{F}}$, where $\mathbb{R}_{\mathcal{F}}$ is the space fuzzy numbers. The following are the derivative inspired by some the differences from the previous section.

Note that $t$-norm of the minimum is not appropriate for the study of the derivative. Intuitively the fuzzy processes (fuzzy functions) have some type of interactivity.

Remark 5.1 When we refer to "limit" for fuzzy function we mean it is continuous in relation to metric $d_{\infty}$. The distance $d_{\infty}: \mathbb{R}_{\mathcal{F}}^{n} \times \mathbb{R}_{\mathcal{F}}^{n} \rightarrow \mathbb{R}_{+} \cup\{0\}$, is defined by

$$
d_{\infty}(A, B)=\sup _{0 \leq \alpha \leq 1} \max \left\{\left|a_{\alpha}^{-}-b_{\alpha}^{-}\right|,\left|a_{\alpha}^{+}-b_{\alpha}^{+}\right|\right\},
$$

where $A$ and $B$ are fuzzy numbers.

The following limits are studied from $d_{\infty}$.

Definition 5.2 [8] Let $F:(a, b) \rightarrow \mathbb{R}_{\mathcal{F}} . F$ is said Hukuhara differentiable (H-differentiable) at $x_{0}$ if the limits

$$
\lim _{h \rightarrow 0^{+}} \frac{F\left(x_{0}+h\right)-{ }_{H} F\left(x_{0}\right)}{h}
$$

and

$$
\lim _{h \rightarrow 0^{+}} \frac{F\left(x_{0}\right)-{ }_{H} F\left(x_{0}-h\right)}{h}
$$

exist and they are equal to some element $F^{\prime}{ }_{H}\left(x_{0}\right)$ that belongs to $\mathbb{R}_{\mathcal{F}} . \quad F^{\prime}{ }_{H}\left(x_{0}\right)$ is called the $H$ derivative of $F$ at $x_{0}$.

Definition 5.3 [11] Let $F:(a, b) \rightarrow \mathbb{R}_{\mathcal{F}} \quad F$ is said generalized Hukuhara differentiable ( $g H$ differentiable) at $x_{0}$ if the limit

$$
\lim _{h \rightarrow 0} \frac{F\left(x_{0}+h\right)-{ }_{g H} F\left(x_{0}\right)}{h}
$$

exists and it is equal to some element $F^{\prime}{ }_{g H}\left(x_{0}\right)$ that belongs to $\mathbb{R}_{\mathcal{F}} . F^{\prime}{ }_{g H}\left(x_{0}\right)$ is called the $g H$-derivative of $F$ at $x_{0}$.

Definition 5.4 [11] Let $F:(a, b) \rightarrow \mathbb{R}_{\mathcal{F}} . F$ is said generalized differentiable ( $g$-differentiable) at $x_{0}$ if the limit

$$
\lim _{h \rightarrow 0} \frac{F\left(x_{0}+h\right)-{ }_{g} F\left(x_{0}\right)}{h}
$$

exists and it is equal to some element $F^{\prime}{ }_{g}\left(x_{0}\right)$ that belongs to $\mathbb{R}_{\mathcal{F}}$. $F^{\prime}{ }_{g}\left(x_{0}\right)$ is called the $g$-derivative of $F$ at $x_{0}$.

Remark 5.5 The gH-derivative and g-derivative generalize the $H$-derivative. Moreover the $g$ derivative generalize the $g H$-derivative. If $\mathrm{H}$ derivative exists, then $g H$ - and $g$-derivative exist and are the same. 
Definition 5.6 [15] Let $F:[a, b] \rightarrow \mathbb{R}_{\mathcal{F}}$, for each $h>0$ sufficiently small, let $J_{h}$ be a joint possibility distribution of $F\left(x_{0}+h\right)$ and $F\left(x_{0}\right)$ with $x_{0} \in[a, b]$. $F$ is said J-differentiable at $x_{0}$ if the limits

$$
\lim _{h \rightarrow 0^{+}} \frac{F\left(x_{0}+h\right)-J_{h} F\left(x_{0}\right)}{h}
$$

and

$$
\lim _{h \rightarrow 0^{+}} \frac{F\left(x_{0}\right)-J_{h} F\left(x_{0}-h\right)}{h}
$$

exist and they are equal to some element $F^{\prime}{ }_{J}\left(x_{0}\right)$ that belongs to $\mathbb{R}_{\mathcal{F}} . \quad F^{\prime}{ }_{J}\left(x_{0}\right)$ is called the $J$ derivative of $F$ at $x_{0}$. At the endpoints of $[a, b]$ we consider only one-sided derivative.

Definition 5.7 [15] Let $F:[a, b] \rightarrow \mathbb{R}_{\mathcal{F}}$, for each $h>0$ sufficiently small, let $F\left(x_{0}+h\right)$ and $F\left(x_{0}\right)$ with $x_{0} \in[a, b]$ be completely correlated fuzzy numbers with joint possibility distribution $C_{h} . F$ is said $C$-differentiable at $x_{0}$ if the limits

$$
\lim _{h \rightarrow 0^{+}} \frac{F\left(x_{0}+h\right)-C_{h} F\left(x_{0}\right)}{h}
$$

and

$$
\lim _{h \rightarrow 0^{+}} \frac{F\left(x_{0}\right)-C_{h} F\left(x_{0}+h\right)}{h}
$$

exist and they are equal to some element $F^{\prime}{ }_{C}\left(x_{0}\right)$ that belongs to $\mathbb{R}_{\mathcal{F}} . \quad F^{\prime}{ }_{C}\left(x_{0}\right)$ is called the $C$ derivative of $F$ at $x_{0}$. At the endpoints of $[a, b]$ we consider only one-sided derivative.

\section{Conclusion}

We studied different versions of difference for fuzzy numbers and compared them in terms of existence (existence means the result is a fuzzy number) and diameter of the resulting $\alpha$-levels. Next we concluded that for a certain epidemiological model the completely correlated difference is more suitable and coherent to reality. Using the differences we define the derivatives. The J-derivative and the $\mathrm{C}$ derivative take into account possible interactivity (dependencies) present in the studied process. It makes sense we use interactivity in fuzzy processes, otherwise we may have a constant function where $F(x+h)-F(x)$ is not zero (as is the case when using the t-norm of the minimum). The derivatives of Subsection 3.1 may be considered interactive, the only one that is not interactive is the Zadeh difference (or standard difference).

\section{Acknowledgment}

The first two authors would like to thank CNPq for financial help (Grants 141085/2014-2 and 305862/2013-8).

\section{References}

[1] L. C. Barros, R. C. Bassanezi, Tópicos em lógica fuzzy e biomatemática, IMECC-Unicamp, Campinas, 2010. (In Portuguese.)

[2] B. Barnabás, L. Stefanini, Generalized differentiability of fuzzy-valued functions, Fuzzy Sets and Systems, 230:119-141 (2013).

[3] Negoita, C. V., and D. A. Ralescu, Application of Fuzzy Sets to System Analysis, ISR Vol. 11., (1975).

[4] V. M. Cabral, Equações diferenciais fuzzy com parâmetros interativos, Doctoral Thesis, in portuguese, IMECC-Unicamp, 2011. (In Portuguese.)

[5] C. Carlsson, R. Fúller and P. Majlender, Additions of completely correlated fuzzy numbers, em IEEE International Conference on Proceedings (Fuzzy Systems), pp.535-539, Julho, Budapest, 2004.

[6] W. A. Lodwick, Constrained interval arithmetic, Technical Report, Center for Computational Mathematics, University of Colorado at Denver, Denver, USA, 1999.

[7] W. A. Lodwick and E. A. Untiedt, A comparison of interval analysis using constraint interval arithmetic and fuzzy interval: analysis using gradual numbers, Annual Meeting of the North American (NAFIPS IEEE), 2008.

[8] M. L. Puri and D. A. Ralescu. Differentials of fuzzy functions, Journal of Mathematical Analysis and Applications, 91.2:552-558 (1983).

[9] L. Stefanini and B. Barnabás, Generalized Hukuhara differentiability of interval-valued functions and interval differential equations, Nonlinear Analysis: Theory, Methods \& Applications, 71.3:1311-1328 (2009).

[10] L. Stefanini, A generalization of Hukuhara difference and division for interval and fuzzy arithmetic, Fuzzy sets and systems, 161.11: 15641584 (2010).

[11] B. Bede, Mathematics of Fuzzy Sets and Fuzzy Logic, Springer-Verlag Berlin Heidelberg, Berlin, Heidelberg, 2013.

[12] L. T. Gomes and L. C. Barros, A note on the generalized difference and the generalized differentiability, 2014, submitted for publication.

[13] L. Edelstein-Keshet. Mathematical models in biology. Vol. 46, Siam, 1987.

[14] J. M. Baetens and B. D. Baets, Incorporating fuzziness in spatial susceptible-infected epidemic models, Proceeding of IFSA-EUSFLAT Conference, Lisbon, Portugal, CD-ROM, 2009.

[15] L. C. Barros and F. S. Pedro, Fuzzy Differential Equations with Interactive Derivative, 2014, submitted for publication.

[16] L. T. Gomes, L. C. Barros and B. Bede. Fuzzy Differential Equations in Various Approaches, Springer, In press. 